NASA Technical Memorandum 106288

\title{
Modeling Void Growth and Movement With Phase Change in Thermal Energy Storage Canisters
}

Douglas Darling, David Namkoong and J. Raymond Lee Skarda Lewis Research Center Cleveland, Ohio

Prepared for the 28th AIAA Thermophysics Conference sponsored by the American Institute of Aeronautics and Astronautics Orlando, Florida, July 6-9, 1993

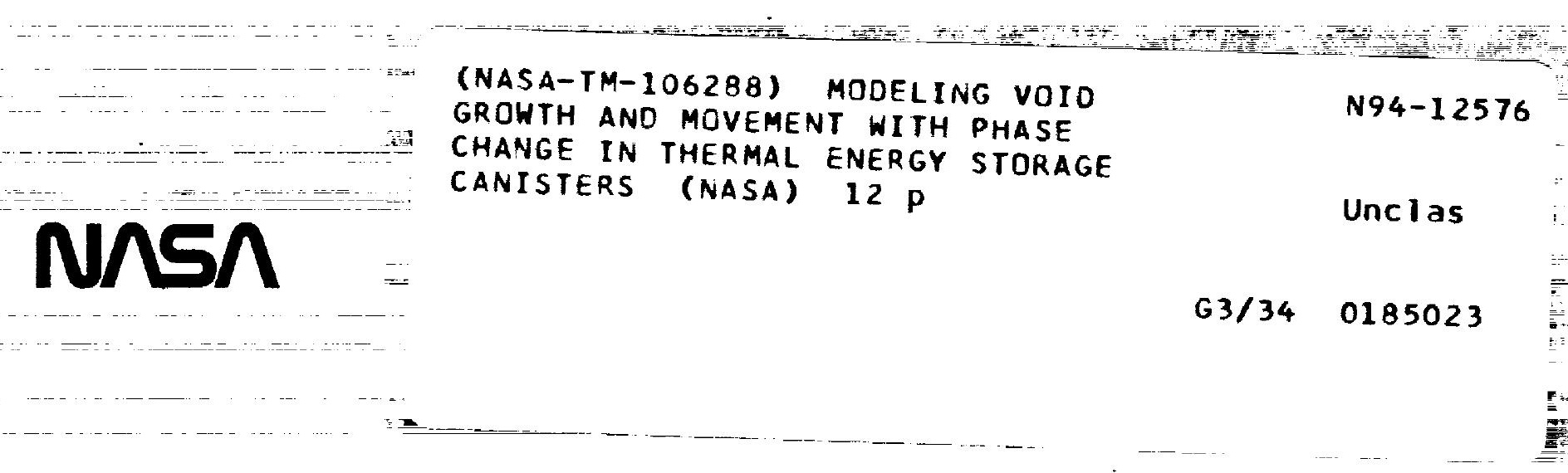


$-\ldots$

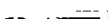

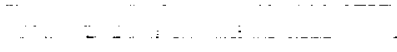

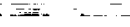



$-$

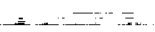

$\therefore \ldots \ldots+\cdots$

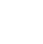

(1) 


\title{
MODELING VOID GROWTH AND MOVEMENT WITH PHASE CHANGE IN THERMAL ENERGY STORAGE CANISTERS'
}

\author{
Douglas Darling David Namkoong J. Raymond Lee Skarda \\ NASA Lewis Research Center \\ Cleveland, Ohio $\mathbf{4 4 1 3 5}$
}

\begin{abstract}
A scheme was developed to model the thermal hydrodynamic behavior of thermal energy storage salts. The model included buoyancy, surface tension, viscosity, phases change with density difference, and void growth and movement. The energy, momentum, and continuity equations were solved using a finite volume formulation. The momentum equation was divided into two pieces. The void growth and void movement are modeled between the two pieces of the momentum equations. Results showed this scheme was able to predict the behavior of thermal energy storage salts.
\end{abstract}

\section{Introduction}

Fluoride salts (e.g. LiF, LiF-CaF $2, \mathrm{NaF}$ ) are common phase change materials for space-based thermal energy storage because their heats of fusion and melting points are suitable for standard cycles and materials. One of the problems with lithium fluoride salts is the large density change they experience during phase change (as much as $30 \%$ ). As the salt freezes voids will form in a thermal energy storage canister due to the density increase. Various problems can arise in the canister depending on the void location. For example, hot spots can develop if the void is located along the canister wall where heat is being added. Or, ratcheting of the canister walls can occur if the salt melts but has no adjacent void to grow into. Thus, it is important to predict where the voids will form in the thermal energy storage canisters.

The position of the void in thermal energy storage canisters is a function of both gravity and surface tension. In the extreme cases the location of the void is obvious. In a 1-g environment the buoyancy will dominate and the void will form at the top of the canister (relative to the direction gravity is acting). In a 0 -g environment sur- face tension will dominate and the void will form along the hottest surface of the canister. However, in a microgravity environment, the effects of surface tension and buoyancy may both be important. In this case the void location is more difficult to predict. This paper describes a scheme for modeling void growth and movement with phase change in microgravity environments.

The NORVEX (NASA-Q Qak Ridge Void EXperiment) computer code was written to model the behavior of phase change material (PCM) in a thermal energy storage (TES) canister $[1,2]$. The geometry and boundary conditions were developed to match those of the Thermal Energy Storage Flight Experiment. During the flight experiment, the phase change material (in this

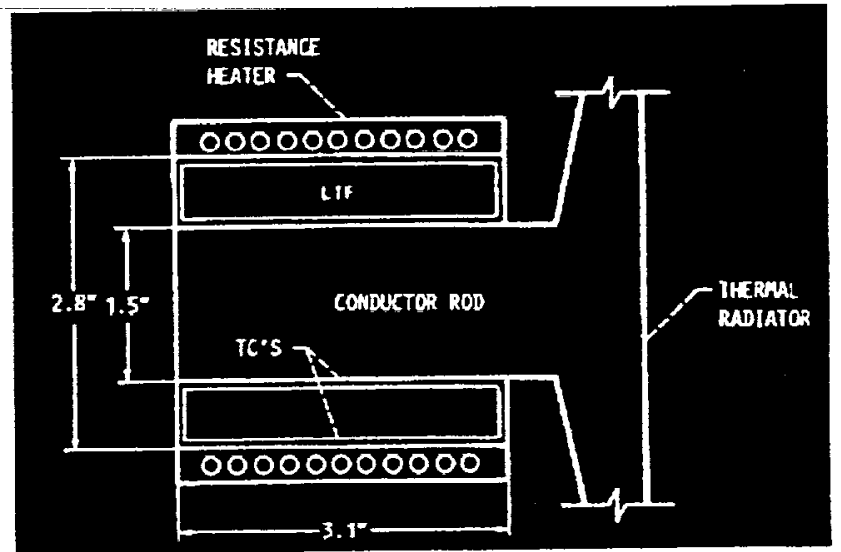

Figure 1 Thermal Energy Storage Flight Experiment hardware

case $\mathrm{LiF}$ ) will be contained in a canister with a truncated right circular cylinder geometry as shown on figure 1. Heat will be added to the outside of the canister to simulate incident flux in a solar dynamic receiver. Heat will be removed from the central core to simulate energy removed by the working fluid of a solar dynamic power system.

\footnotetext{
${ }^{1}$ Copyright $\odot 1993$ by the American Institute of Aeronautics and Astronautics, Inc. No copyright is asserted in the United States under Title 17, U.S. Code. The U.S. Government has royalty-free license to exercise all rights under the copyright claimed herein for Govemmental purposes. All other rights are reserved by the copyright owner.
} 
The following capabilities are required to model TES thermal hydrodynamic behavior:

Model $1 \mathrm{-g}, 0 \mathrm{~g}$, and microgravity conditions.

Track the phase change front.

Predict void growth and movement.

Include buoyancy, surface tension, and viscous effects.

Solve continuity, momentum, energy equations in the

phase change material (PCM).

Predict conduction in canister, core, flare.

The purpose of this paper is to describe the problems and solutions of modeling void growth and movement with phase change in microgravity environments. The principle application of this work is to model thermal energy storage media used in solar dynamic power systems.

\section{Basic Characteristics of Algorithm}

The canisters to be used in the first two Thermal Energy Storage Flight Experiments (TES-1 \& TES-2) will be truncated cylinders as pictured in figure 1 . During the flight experiments heat will be periodically added to the outside of the canister to simulate solar flux entering a solar receiver during the sunlit portion of an orbit. Heat will be removed from the inside radius of the canister to simulate heat being removed by the working fluid in a solar dynamic power system. The geometry in NORVEX is setup to match these canisters as shown in figure 2 . The canisters to be used in the flight experiments have the following dimensions:
$\mathrm{rl}=\quad 1.80 \mathrm{~cm}$
$\mathrm{r} 2=1.90 \mathrm{~cm}$
$\mathrm{r} 3=3.48 \mathrm{~cm}$
$\mathrm{r} 4=3.58 \mathrm{~cm}$
$\mathrm{z} 1=0.00 \mathrm{~cm}$
$\mathrm{z} 2=0.10 \mathrm{~cm}$
$\mathrm{z} 3=6.99 \mathrm{~cm}$
$\mathrm{z} 4=\quad 7.09 \mathrm{~cm}$

A cylindrical coordinate system is used. No-slip and no-penetration boundary conditions are used at the top, bottom, inner radius, outer radius of the canister. A periodic boundary condition is used in the azimuthal direction. NORVEX is set up to accept either a heat flux or a temperature boundary condition on each surface of the canister.

In the NORVEX computer code, conservation laws are applied to finite volume cells. Each cell can contain all liquid, all solid, all void, or any combination of solid,

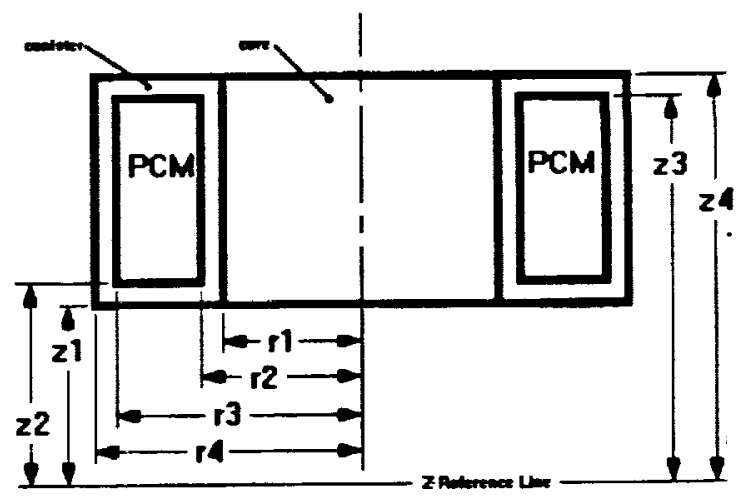

Figure 2 Overall geometry of canister used in NORVEX

liquid, and void. The quality of each finite volume cell is tracked by the fluid fraction (one minus the void fraction) and the liquid fraction. These are treated as state variables, applied to each cell. The position of the free surface is located in cells with a fluid fraction between zero and one. The orientation of the free surface is tracked by the gradient of the fluid fraction. This is similar to the volume of fluid (VOF) of Hirt and Nichols [3]. In addition, a marker is placed in each cell to indicate if there is a free surface (marker set to 1 for cell with surface, 0 for completely full or completely empty cells). This is redundant information, knowing the fluid fraction should be enough. However, these markers can be used to avoid many IF statements when determining the surface forces and avoiding averaging across the surface (derivatives should not be assumed continuous across the surface). Avoiding many IF-statements makes the program run faster. Figure 3 shows the definition of a general finite volume cell, used in NORVEX. The state variables, such as temperature, pressure, liquid fraction, etc. are defined at the center of each cell. The velocities are defined at the faces of the cells. Each velocity component is defined at the face perpendicular to it.

The energy equation is solved by balancing energy leaving, entering, and being stored within each finite volume cell. Energy is transferred in and out of each cell by both conduction and advection. The energy stored in each cell is represented by the enthalpy method. This way the latent heat and sensible heat can be stored the same way, as enthalpy. The temperature of each cell is calculated for each cell knowing the enthalpy using constitutive relations. A complete description 


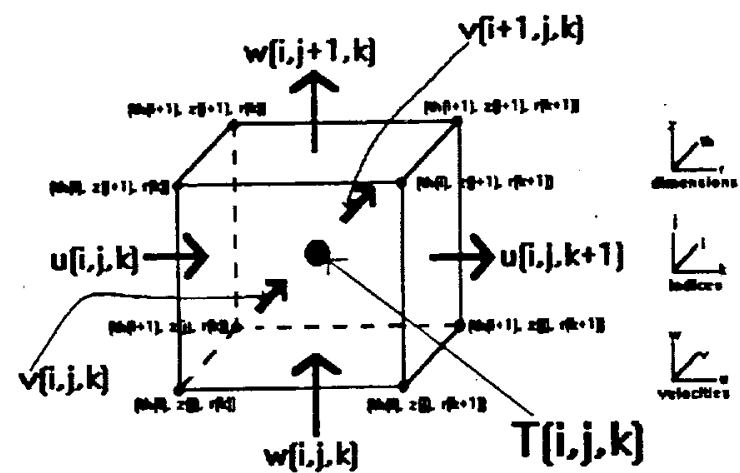

Figure 3 Finite volume definition used in NORVEX

of the energy equations used in NORVEX is given by Drake [4].

The momentum equation includes buoyancy and viscous effects. The Boussinesq approximation is used to model buoyancy. The flow will always be laminar for conditions in the thermal energy storage canisters under consideration, so no turbulence model is included [5]. Surface tension and pressure boundary conditions are included for finite volume cells which contain a free surface. These boundary conditions will be discussed in detail in the next section.

The momentum equation is solved in two parts. The dicretized time derivative of velocity in the momentum equation is split into two pieces, as shown in equation 1.

$$
\rho \frac{\overline{V^{n+1}}-\overline{V^{n}}}{\Delta t}=\rho \frac{\overline{V^{n+1}}-\overline{V^{*}}}{\Delta t}+\rho \frac{\overline{V^{*}}-\overline{V^{n}}}{\Delta t}
$$

Each part of the momentum equation uses part of this time derivative. The first part of the momentum equation contains viscosity, gravity, and advection terms. The second part of the momentum equation contains the gradient of the pressure.

1st Part of Momentum Equation:

$$
\rho \frac{\overline{V^{*}}-\overline{V^{n}}}{\Delta t}=\operatorname{div}(-\rho \bar{V} \bar{V}+\bar{\tau})+\rho \bar{I}
$$

2nd Part of Momentum Equation:

$$
\rho \frac{\overline{V^{n+1}}-\overline{V^{*}}}{\Delta t}=-\operatorname{grad}(P)
$$

At a given time step, all quantities in the first part of the momentum equation except $\mathrm{V}^{*}$ can be calculated from know quantities or expressed in terms of $\mathrm{V}^{*}$. Thus, the first part of the momentum equation can be solved directly for $\mathrm{V}^{*}$. The second part of the momentum equation, however, has two unknowns, $P$ and $V^{n+1}$. But, $V^{n+1}$ can be eliminated from the equation by taking the divergence of the second part of the momentum equation.

$$
\frac{\operatorname{div}\left(\rho \overline{V^{n+1}}\right)}{\Delta t}-\frac{\operatorname{div}\left(p \overline{V^{*}}\right)}{\Delta t}=-\operatorname{div}(\operatorname{grad}(P))
$$

Then, the continuity equation can be used determine a value for $\operatorname{div}\left(\rho \mathrm{V}^{n+1}\right)$. A detailed description of this solution of the momentum equation see Wichner, et al. [1] and Drake [4].

In NORVEX, the $\operatorname{div}\left(\rho \mathrm{V}^{\mathrm{n}+1}\right)$ term represents the rate that mass is either entering or leaving a finite volume cell. For most finite cells, the $\operatorname{div}\left(\rho \mathrm{V}^{\mathrm{m}+1}\right)$ is simply zero (since the liquid and solid are incompressible). However, a cell with a free surface may lose or gain mass from one time step to the next. In addition, finite volume cell undergoing phase change will lose or gain mass if the densities of the solid and liquid phases are different. Thus, the value of $\operatorname{div}\left(\rho \mathrm{V}^{n+1}\right)$ needs to be coupled with the energy and momentum equations. This coupling will be described in detail in section 4 .

The basic sequence of a typical NORVEX time step is as follows: update time dependent boundary conditions, solve the energy equations for temperature, solve 1st part of momentum equation (viscous, surface tension, buoyancy, and advect.jve terms), determine void growth or shrinkage based on phase change, predict void movement based on solution of 1st part of the momentum equation correct results of 1 st momentum equation with pressure equation, this is also where continuity is be satisfied, then go to next time step or quit. 
The energy equation is solved before the momentum equation. Velocities from the previous time step are used in the advective terms of the energy equation, since there is no global iteration between momentum and energy equations. As a result, the energy associated with the mass flow predicted by the momentum equations will be accounted for in the energy equation on the following time step.

\section{Boundary Conditions}

The z-momentum equation in cylindrical coordinates is given in equation 5 (before the Boussinesq approximation was added) [6].

$$
\rho \frac{\partial w}{\partial t}+\rho u \frac{\partial w}{\partial I}+\frac{\rho v}{I} \frac{\partial w}{\partial \theta}+\rho w \frac{\partial w}{\partial z}=-\frac{\partial P}{\partial z}+\rho g
$$

$$
+\mu\left[\frac{1}{I} \frac{\partial}{\partial I}\left(r \frac{\partial W}{\partial I}\right)+\frac{1}{I^{2}} \frac{\partial^{2} W}{\partial \theta^{2}}\right]+f_{z}
$$

Gravity and pressure effects are included in this equation. However, surface tension is not expressed because it is a boundary force rather than a body force. Obviously, it is a boundary condition. However, it is a difficult boundary condition to formulate since the boundary may be moving and may or may not be orientated parallel to a coordinate axis. For example, the shear stress caused by variations in surface tension with temperature could be formulated as follows for an analytical solution.

$$
\mu \frac{\partial V_{s}}{\partial n}=\left(\frac{\partial \sigma}{\partial T}\right) \nabla T_{s}
$$

where, $\partial V_{s} / \partial n$ is the derivative of the velocity component along the surface in the direction of the outward normal of the surface, $\sigma$ is the surface tension, and $\nabla T_{s}$ is the temperature gradient projected onto the surface. This would be a difficult boundary condition to implement if the surface is far from being parallel to one of the coordinate axes.

However, in a finite volume model, this boundary condition is a little easier to model. It can simply be added as a force to each cell that contains a free surface. The orientation of the free surface can be determined from the fluid fraction. The outward unit normal of the free surface is given by equation 8 .

$$
\bar{n}=-\frac{\nabla(\text { fluid fraction })}{\nabla(\text { fluid fraction }) \mid}
$$

The projection of the temperature gradient on the free surface can be calculated by equation 9 .

$$
\nabla T_{s}=\nabla T-(\nabla T \cdot \bar{n}) \bar{n}
$$

The variation in surface tension is a material property that is input by the user in NORVEX.

$$
\gamma=\frac{d \sigma}{d T}
$$

Finally, the force per unit area of free surface can be calculated by equation 11 .

$$
F_{\varepsilon}=\gamma \nabla T_{s}
$$

This force is added as a source term in the first part of the momentum equation in NORVEX.

- Similarly, the surface boundary condition caused by the curvature of the free surface can be expressed as in equation 12 , where $K$ is the curvature of the surface.

$$
P_{p a m, \text { surface }}=P_{\text {void }}-2 \sigma \mathrm{K}
$$

And, the boundary condition for wetting can be added as a force to cells with a free surface that are next to the wall of the canister, as in equation 13 , where $L$ is the length the surface contacts a wall in the finite volume cell.

$$
F_{\text {wet }}=\sigma L \cos (\operatorname{con} \text { tact angle) }
$$

Velocity boundary conditions are straight forward. A no slip boundary condition is used at the solid walls. This is implemented by creating an imaginary cell on the other side of the solid wall with a velocity in the opposite direction (but the same magnitude) as the boundary cell. This opposite velocity is then used to calculate the viscous forces for the boundary cell. In addition, a no penetration boundary condition is used at the walls and the solidliquid interface. 
Finally, a pressure boundary condition needs to be included at the free surface. Physically, the pressure at the free surface should be the pressure of the void (plus any surface curvature effect). However, the "pressure" predicted in NORVEX is not the actual pressure. Since the Boussinesq approximation is used in the momentum equation, the pressure that is calculated is the real pressure minus the hydrostatic contribution. Thus, the pressure assigned to the free surface must also be adjusted by the hydrostatic contribution. This is very important when the free surface is moving due to gravity. The correction for the pressure boundary condition is calculated by determining the height $(\mathrm{h})$ of the surface relative to some datum in the gravity direction. The height of the surface in each cell is calculated in NORVEX by projecting the location of the free surface for that cell onto the gravity vector. Then the correction to the pressure boundary condition is simply $\rho_{\text {liguid }} g h_{\text {surface }}$. This correction is then added to the cells containing a free surface.

\section{Void Growth and Movement}

In NORVEX, the void growth and movement calculations are performed after the first part of the momentum equation is solved. The results of the void growth and movement calculations are values of mass gain or loss for each cell $\left(\partial \mathrm{m}_{\text {cell }} / \partial t\right)$. This will be substituted for the $\operatorname{div}\left(\rho V^{n+1}\right)$ term in the second part of the momentum equation using continuity (equation 14).

$$
V O I_{c e l l} \operatorname{div}\left(\rho V^{n+1}\right)_{c e l l}=-\frac{\partial m_{c e I I}}{\partial t}
$$

The second part of the momentum equation will then predict the velocities necessary to support the predicted void growth or movement. In order for the void movement to be coupled correctly with the momentum equation, all forces that affect void position must be included in the first part of the momentum equation. This includes surface tension forces as well as the correction of the surface pressure for hydrostatic effects. (Note: the "pressure" calculated in the second part of the momentum equation will still be the real pressure minus the hydrostatic contribution.)

Most finite volume cells have no net mass loss or addition during a given time step. For those cells $\partial \mathrm{m}_{\text {cell }} / \partial t$ is simply set to zero, since the liquid and solid are both incompressible. However, cells with a free surface may have a net change in mass as the surface moves. In addition, cells undergoing phase change may have a net increase or decrease in mass if the densities of the solid and liquid are not the same.

Cells in which PCM is solidifying will have an increase in mass if the solid density is greater than the liquid density. This is due to the liquid pulled into the cell as the PCM shrinks when solidifying. However, if a cell solidifies, but the solid density was less than the liquid density, the cell would lose mass as the PCM expands. This is similar to a phase change velocity. The rate that mass is being drawn in or pushed out of a cell undergoing phase change can be calculated from equation 15.

$$
\frac{\partial m_{\text {ceII }}}{\partial t}=\frac{\partial m_{\text {solidd }}}{\partial t}\left(1-\frac{p_{\text {liquid }}}{p_{\text {solid }}}\right)
$$

The rate of change of the solid mass in the cell is known from the solution of the energy equation energy equation.

The case of $\rho_{\text {solid }}>\rho_{\text {liquid }}$ is important for NORVEX, because all thermal energy storage salts being considered have higher solid densities than liquid densities. A special problem arises in finite volume cells when the - solid density is larger than the liquid density. The problem has to do with allowing the PCM in a cell to become completely frozen while the cell remains completely full. During the time step that all the PCM in a cell becomes completely solid, it will shrink (just like always), drawing in enough liquid to keep the cell full. Then, this new liquid will solidify, but now it too shrinks and the cell is no longer full so it needs to draw in more liquid. This new liquid also solidifies, shrinks and requires even more liquid. Without a check this process would continue indefinitely, which is physically unrealistic. The cell will never be completely full of solid. The solution to this problem is to alter the $\partial \mathrm{m} / \partial \mathrm{t}$ specified for the cell for this situation. After the energy equation is performed, NORVEX checks the enthalpy of each cell that has become completely solid on that time step. If the enthalpy is low enough to solidify any saturated liquid entering the cell then the net mass flow into the cell is prescribed by equation 16 (rather than equation 15).

$$
\frac{\partial m_{c e 11}}{\partial t}=\frac{\partial m_{\text {solid }}}{\partial t}\left(\frac{\rho_{\text {solid }}}{\rho_{\text {liquid }}}-1\right)
$$

Using equation 16 , the cell will be completely full and solid on the following time step. 
It is important to keep track of the change in mass of the solid when doing these calculations rather than changes in liquid fraction. Both the mass of the solid and the liquid fraction change while the energy equations are solved. But then, the mass of the solid is a constant quantity between energy calculations, while liquid fraction may not be. For example, as we have seen, liquid will be drawn into a cell as the PCM solidifies (for $\rho_{\text {solid }}>\rho_{\text {liquid }}$. So, at the end of the flow calculation there is will be more liquid in the cell than at the beginning of the flow calculation, while the amount of solid remains constant. Thus, the liquid fraction is greater at the end of the flow calculation. While liquid fraction is a useful state variable in many situations (particularly when $\rho_{\text {solid }}=\rho_{\text {liquid }}$ ), it is difficult to use to conserve mass in this case.

Most of the forces which dictate void movement are included in the first part of the momentum equation. This includes, surface tension, viscous forces, inertia, and the additional pressure force due to the variation of the height of the surface relative to gravity, to make up for the hydrostatic pressure being subtracted for the Boussinesq approximation. The assumption used in NORVEX is these forces have more of an influence on the motion of the free surface than the "pressure" predicted in the second par of the momentum equation. This assumption holds tue for all of the cases discussed in section 5, since the predicted pressures represented much smaller forces on the surface cells than the surface tension, etc. This "pressure" was still important, however, to satisfy continuity within the liquid PCM.

Given this assumption, the $V^{*}$ 's (predicted in the first part of the momentum equation) tell us where the surface would like to move if it was not restricted by continuity. But, the cells containing the surface are, of course, subject to continuity, so it needs to be addressed.

For each cell containing a free surface, a desired change in mass is calculated from the solution of the first part of the momentum equation.

$$
\left(\frac{\partial m_{c e 11}}{\partial t}\right)_{\text {vant }}=-V o I_{c e l 1} \operatorname{div}\left(\rho \overline{V^{*}}\right)
$$

If the surface cell is also undergoing phase change, then the contribution from phase change, described above, is also included (see equations 15 and 16). Each cell is subject to obvious constraints: a cell cannot have negative mass (lower limit $\left.=(\partial \mathrm{m} / \partial \mathrm{t})_{\min }\right)$, and a cell cannot be overfilled (upper limit $\left.=(\partial \mathrm{m} / \partial t)_{\mathrm{max}}\right)$. If the $\mathrm{V}^{*}$ 's predict a cell to have negative mass, NORVEX will assign that cell a $\partial \mathrm{m} / \partial \mathrm{t}$ such that it mass will become zero. Then it looks for an adjacent cell that can make up the difference. Similarly, if the $V^{*}$ 's predict a cell to become over filled, NORVEX will assign a $\partial \mathrm{m} / \partial \mathrm{t}$ such that the cell will be completely full and again looks for an adjacent cell to make up the difference. This enables the free surface to move from one cell to another. The final constraint is the total mass in the canister cannot change. So, equation 18 must be satisfied.

$$
\sum \frac{\partial m_{c o 11}}{\partial t}=0
$$

The mass is redistributed to satisfy equation 18 by interpolating between either $(\partial \mathrm{m} / \partial \mathrm{r})_{\mathrm{mant}}$ and $(\partial \mathrm{m} / \partial \mathrm{t})_{\max }$ or between $(\partial \mathrm{m} / \partial t)_{\text {want }}$ and $(\partial \mathrm{m} / \partial t)_{\min }$ whichever is required to get the sum of all $\partial \mathrm{m} / \partial \mathrm{t}$ 's to be zero. This is a rather coarse way to redistribute the mass. However it preserves the relative importance of each force that makes the voids move, it ensures mass will be conserved, and it is typically needed only when a cell becomes completely empty or completely full and there is no suitable adjacent cell to take up the slack. This redistribution of - mass is one point to be targeted for improvement after the results are obtained from the Thermal Energy Storage Flight Experiments.

\section{Results}

Most of the capabilities described in the previous section have been successfully implemented in NORVEX. The exceptions are the normal surface tension boundary condition and wetting. In this section we will look at some NORVEX predictions to compare the various forces which drive the liquid motion and the void motion.

First we will compare the effects of tangential surface tension force with buoyancy forces. To demonstrate these effects several cases were run with NORVEX. In all cases gravity was directed in the positive z-direction, and heat was added to the outer radius of the canister at a rate of $250 \mathrm{~W}$, while all other sides were adiabatic. NORVEX was initialized with all liquid in the canister, and a void at the top (relative to gravity) of the canister.

For the first case, only buoyancy was included. A coefficient of volumetric expansion of $2.7 \times 10^{-6} \mathrm{~K}^{-1}$ and a 


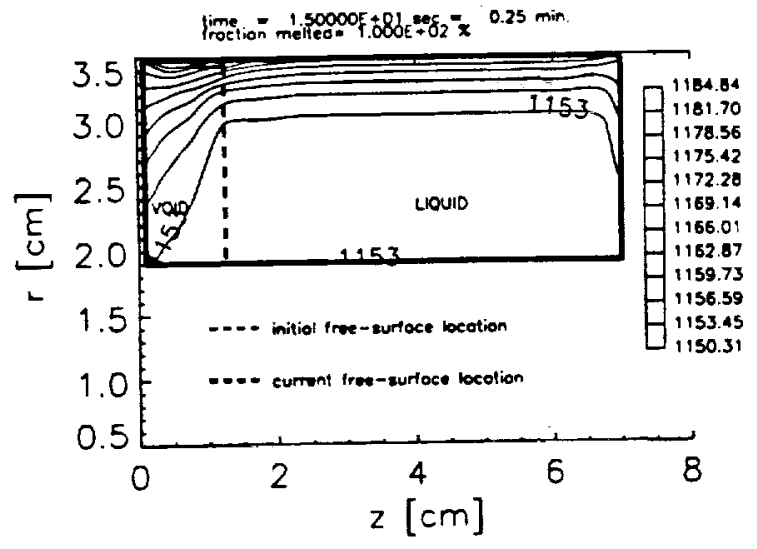

Figure 4 Temperatures, buoyancy only, $0.1 \mathrm{~g}$

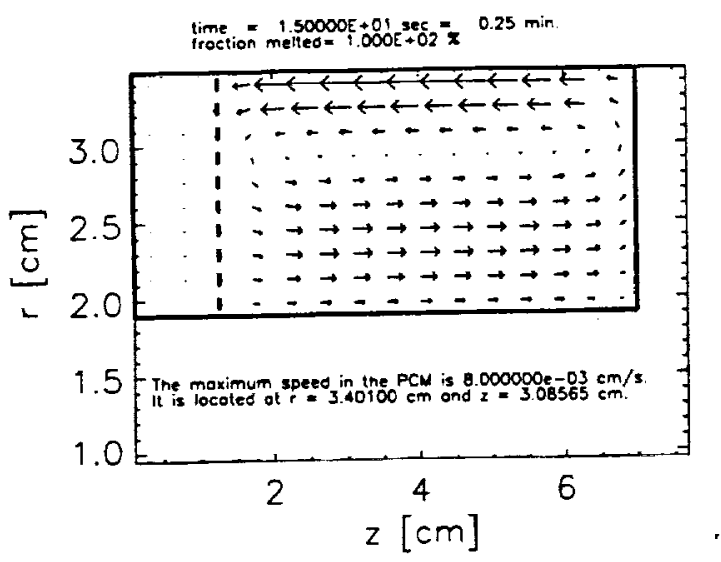

Figure 5 Velocities, buoyancy only, $0.1-\mathrm{g}$

gravity environment of $0.1 \mathrm{~g}$ were used. In this case surface tension forces were not included. Figures 4 and 5 show temperature and velocity predictions for this case at time $=15 \mathrm{sec}$. These plots represent a cross section of the canister. The velocities were fairly low in this case. The maximum velocity wa only $0.008 \mathrm{~cm} / \mathrm{s}$. This small velocity had little effect on the heat transfer. The temperature distribution was similar to what one would expect for straight conduction (including conduction in the canister walls). In addition, free convection had no noticeable effect on the location of the free surface.
Next a tangential surface tension force was included in the model. A value for the change in surface tension with temperature of -233 dynes/cm-K was used. Figures 6 and 7 show temperature and velocity predictions at time $=15 \mathrm{sec}$. The velocities were much larger with the surface tension forces than without. The speed of the phase change material was as high as $1.3 \mathrm{~cm} / \mathrm{s}$. The flow also had a much larger effect on the temperature distribution, particularly near the free surface, where the flow was greatest. However, the free surface only moved very slightly from its initial location. So, even though the surface tension force produced a much larger flow than gravity-driven buoyancy, tangential surface tension still could not compete with gravity to locate the free surface.

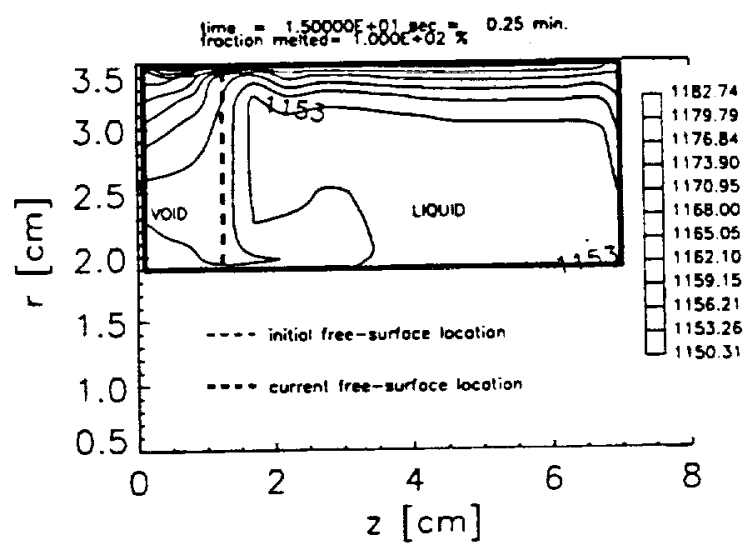

Figure 6 Temperatures, surface tension added, $0.1-g$

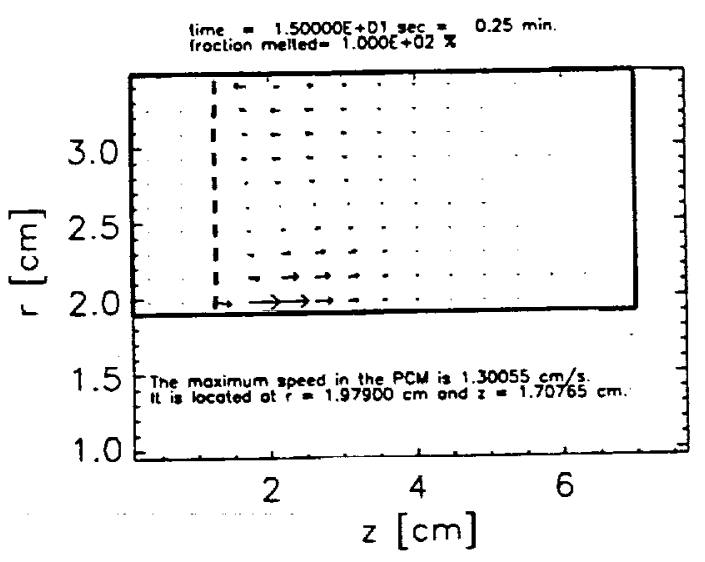

Figure 7 Velocities, surface tension added, $0.1-g$ 
An important conclusion about averaging can be drawn from figure 7 . There is a velocity vector plotted at the lower end of the free surface which appears to be coming out of the free surface. This was not really predicted by NORVEX. It is a result of the averaging performed by the graphics routine. The graphics routine has no way of knowing it should not average across the free surface. This should serve to illustrate the unrealistic results obtained when averaging is performed across a discontinuity. NORVEX checks for the free surface using its markers, therefore does not take averages or derivatives across it.

To demonstrate that gravity will indeed move the surface to this position, another case was run. This case used identical properties as the previous case (still 0.1 g). However, the free surface was initialized in a nonequilibrium position. A hump was included on the free surface, then NORVEX was allowed to predict the movement of the free surface by gravity and surface tension. As shown in Figures 8 and 9, the free surface tended toward the expected equilibrium position. After 15 seconds, the oscillations of the free surface had damped out and the flow looked much the same as the previous case, except there was slightly more salt due to the extra hump added to the free surface.

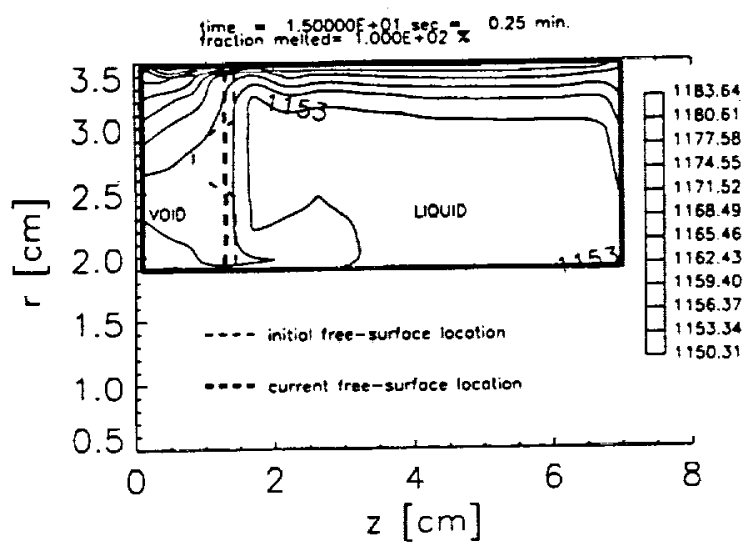

Figure 8 Temperatures, initial hump on surface, $0.1-g$

Eventually, if the gravity was reduced low enough, the tangential surface tension had a noticeable an effect on the position of the free surface. A case was run with tangential surface tension forces as before, but gravity was reduced to $0.01 \mathrm{~g}$. After 15 seconds of simulation, the tangential surface tension has pulled PCM slightly off the hot outer radius toward the cooler inner radius as shown on figure 10.



Figure 9 Velocities, initial hump on surface, $0.1-\mathrm{g}$

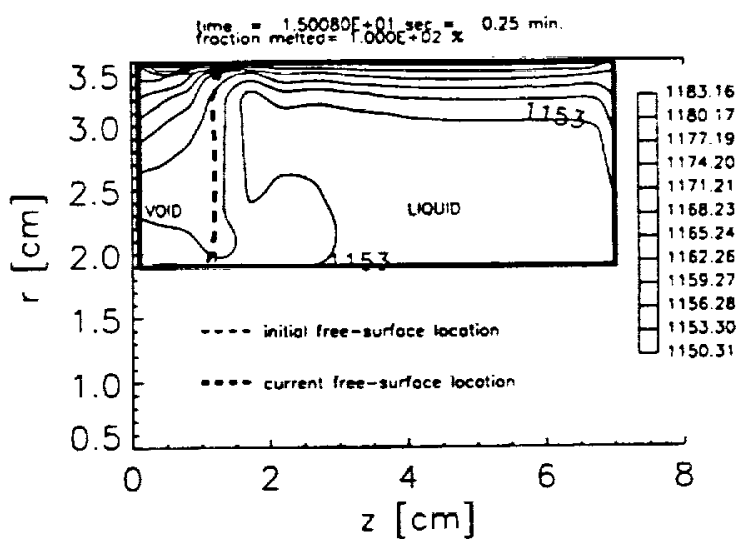

Figure 10 Temperatures, $0.01-\mathrm{g}$

Next, NORVEX was run for phase change without a density difference $\left(\rho_{\text {solid }}=\rho_{\text {liquid }}\right.$ ). A constant heat flux boundary condition was used on the canister. Heat was removed from the canister at $450 \mathrm{~W}$, distributed evenly over the outer radius of the canister, all other sides were adiabatic. Gravity acted in the positive z-direction at 1 g. Figures 11 and 12 show temperature distributions within the canister after 225 seconds and 475 seconds respectively. Early in the solidification, the flow of the liquid PCM affected the shape of the solid-liquid front. The liquid flowed in a clockwise direction at time $=225$ seconds, due to both free convection and tangential surface tension. As a result, the solid tended to get thicker farther down the canister (relative to gravity). 
But, by 475 seconds, the liquid PCM was almost entirely at the phase change temperature. Thus, there was no temperature gradient in the liquid to drive either free convection or surface tension driven convection. The only force, at this point, to control the location of the free surface was gravity (it was dominant in this case from the beginning). At this point, movement of the phase change front was controlled almost entirely by conduction through the solid PCM and canister walls. As a result, the phase change front moved more quickly near the void. This makes sense since the heat flux boundary condition was applied evenly over the outside radius of the canister, including where the void was along the canister wall.

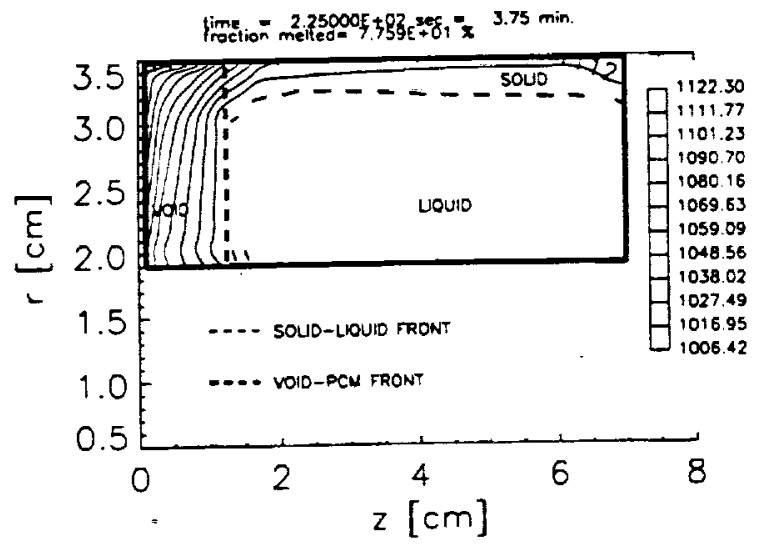

Figure 11 Temperatures for phase change in $1-\mathrm{g}$ at $225 \mathrm{~s}$.

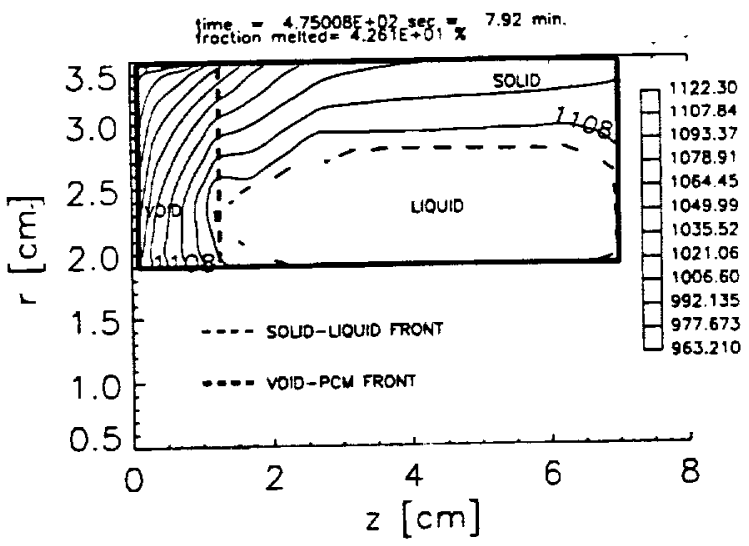

Figure 12 Temperatures for phase change at $1-g$ at $475 \mathrm{~s}$.
Finally, NORVEX was run to demonstrate its ability to model phase change with a density difference. The solid density was $2.19 \mathrm{~g} / \mathrm{cm}^{3}$, while the liquid density was $1.79 \mathrm{~g} / \mathrm{cm}^{3}$. A void was, again, initialized at the top of the canister relative to gravity. Gravity acted in the positive z-direction at 1-g. Heat was removed from the end opposite the void. Figures 13 and 14 show the predicted velocity distributions at time $=10 \mathrm{sec}$ and time $=140 \mathrm{sec}$ respectively. This case is interesting, because free convection is dominant over surface tension driven flow. The free convection is established as heat is conducted along the canister walls. However, surface tension had almost no effect, because there is almost no temperature variation along the surface. Also, as the PCM solidified, the free surface moved. As it moved, the free surface always remained normal to gravity, as expected for 1-g.

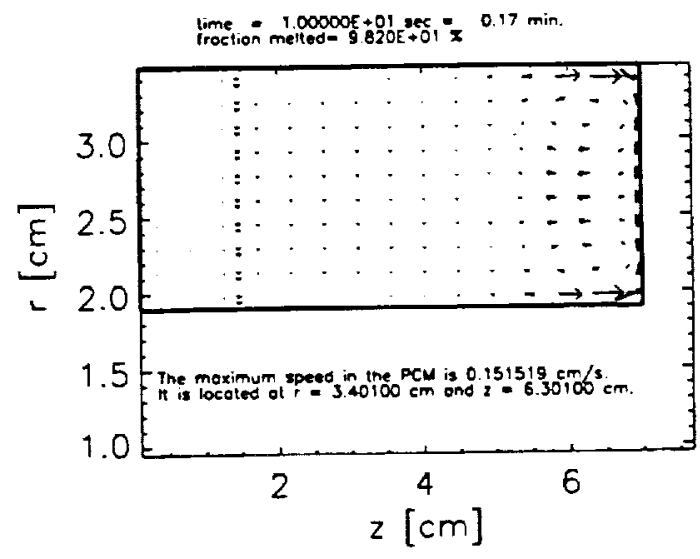

Figure 13 Phase Change, $\rho_{\text {solid }}>\rho_{\text {liquid }}$

This discussion does not represent a new understanding of the physics. It is simply to show that NORVEX can model these situations. But, as mentioned above, the normal surface tension and wetting forces are not yet included in NORVEX. The normal forces should not effect the cases discussed here very much since gravity was always dominant in the position of the free surface and its curvature was small (although wetting may have an effect). However, if the gravity environment was reduced even further, the free surface would move even farther, the free surface would move even farther, and the normal component of surface tension would be the dominant means of holding the PCM in one piece. Once these forces are added NORVEX should be able to model much lower gravity conditions. 


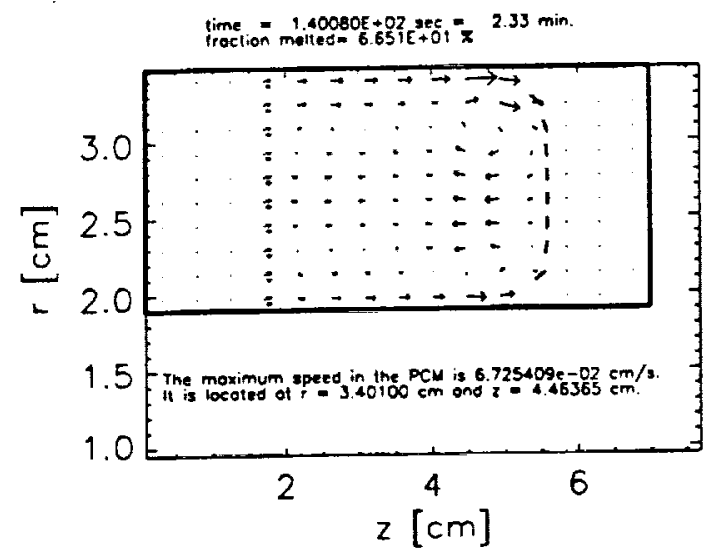

Figure 14 Phase change, $\rho_{\text {solid }}>\rho_{\text {liquid }}$

\section{Conclusion}

We have described a scheme to model the thermal hydrodynamic behavior of phase change material in thermal energy storage canisters with void growth and movement. At this time NORVEX can model the behavior of phase change material as long as gravity is still important for controlling the location of the free surface. Phase change with and without density change can be modeled. The model in NORVEX includes tangential surface tension, buoyancy, and viscosity forces. However, normal surface tension forces are needed to simulate very low gravity cases. NORVEX, particularly the void movement model, will be improved when the results of the Thermal Energy Storage flight experiment have been obtained.

\section{References}

1. Wichner, R. P., A. D. Solomon, J. B. Drake, and P. T. Williams, "Thermal Analysis of Heat Storage Canisters for a Solar Dynamic Space Power System," ORNL-TM-10665. 1988.

2. Wilson, D. G., R. E. Flanery, "Modeling Cyclic Melting and Refreezing in a Hollow Metal Canister," ORNL-6497, 1988.

3. Hirt, C. W. and B. D. Nichols, "Volume of
Fluid (VOF) Method for the Dynamics of Free Boundaries," Journal of Computational Physics, Vol. 39, pp. 201-225, 1981.

4. Drake, J. B., "Modeling Convective Marangoni Flows with Void Movement in the Presence of Solid-Liquid Phase Change," ORNL6516, DE90 006474, 1990.

5. Skarda, J. Raymond, David Namkoong, and Douglas Darling, "Scaling Analysis Applied to the NORVEX Code Development and Thermal Energy Flight Experiment," AlAA91-1420, 1991.

6. Bird, R. Byron, Warren Stewar, Edwin Lightoot, Transport Phenomena, John Wiley and Sons, p. 85, 1960. 



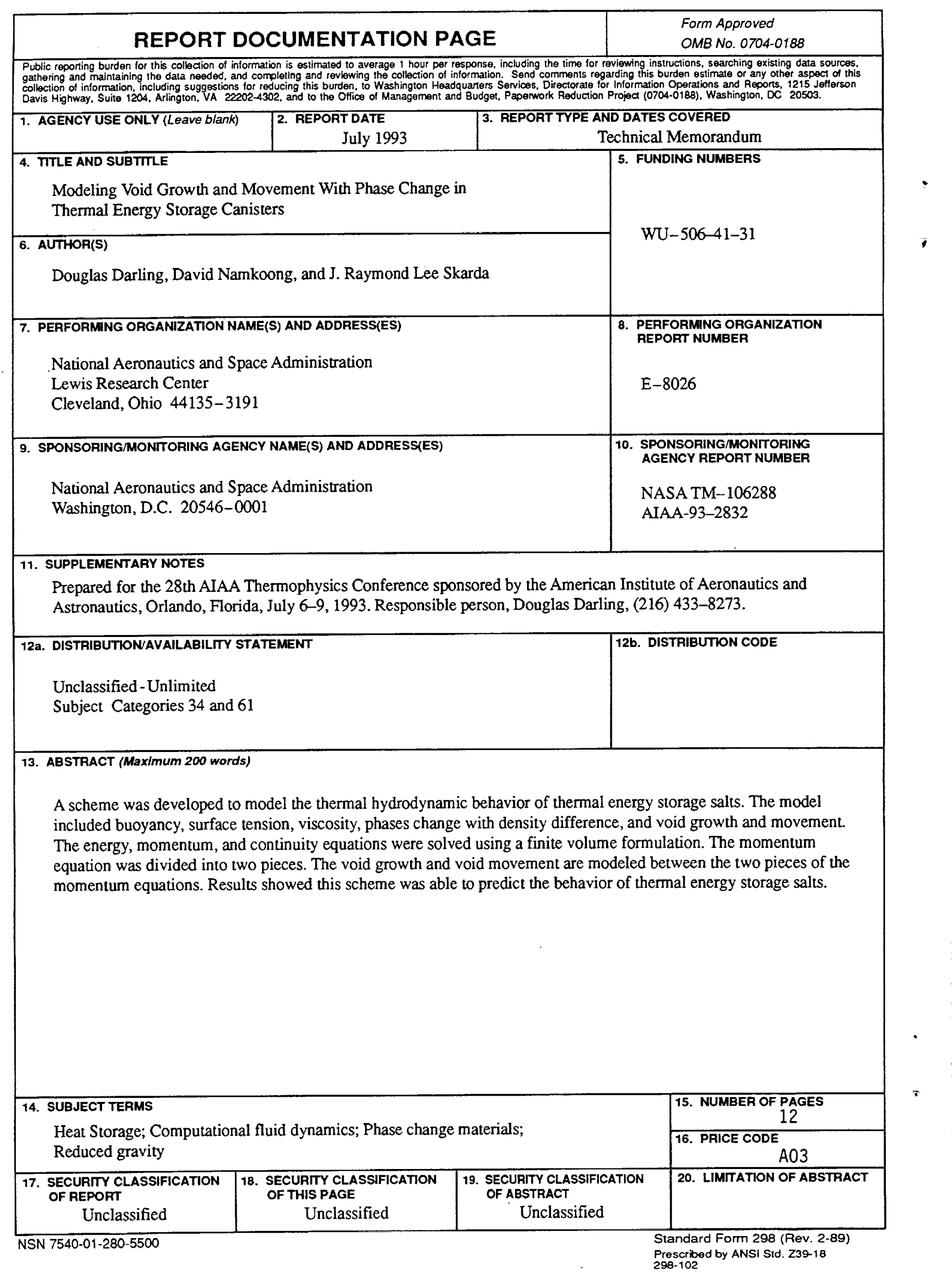

